\title{
PROCESOS ARTESANALES PARA LA PRODUCCIÓN SOSTENIBLE DE TABLEROS DE CAÑA FLECHA (GYNERIUM SAGITTATUM)
}

\section{HANDCRAFT PROCESS FOR SUSTAINABLE PRODUCTION OF ARROW CANE PARTICLE BOARDS (GYNERIUM SAGITTATUM)}

\author{
Pedro Arturo Martínez Osorio, M.Sc. (UNESP - CECAR) \\ Paula Da Cruz Landim, PhD. (UNESP) \\ Tomás Queiroz Ferreira Barata, PhD. (UNESP) \\ Palabras Clave \\ Design; Artesanado; Paneles de partículas; Gynerium sagittatum.
}

\section{Key Words}

Design; Handcraft; Particle boards; Gynerium sagittatum.

\section{RESUMEN}

Se presentan resultados parciales de un estudio que relaciona artesanado y design, con el objetivo de reinterpretar técnicas y conocimientos indígenas, para ser aplicados al desarrollo de productos y procesos más sostenibles con Gynerium sagittatum. Se identifican aspectos interesantes en los procesos artesanales de diferentes comunidades de artesanos, especialmente de las comunidades indígenas Zenú de Colombia, para ser aplicados en el procesamiento sostenible de tableros de partículas de Gynerium sagittatum. Algunos aspectos deben ser mejorados para la obtención de un producto competitivo que cumpla los requisitos del mercado para su uso en diversos sectores, especialmente en el sector de mobiliario en madera.

\section{ABSTRACT}

Partial results of a study that relates craft and design are presented, with the objective of re-interpreting indigenous techniques and knowledge, to be applied to the development of more sustainable products and processes in Gynerium sagittatum. Interesting aspects are identified in the artisanal processes of different artisan communities, especially from the Zenú indigenous communities of Colombia, to be applied in the sustainable processing of particle boards from Gynerium sagittatum. Some aspects must be improved to obtain a competitive product that meets market requirements for use in various sectors, especially in the wood furniture sector. 


\section{INTRODUCCIÓN}

Este trabajo presenta resultados parciales de una investigación en curso que aborda la relación entre design y artesanado, con el objetivo de desarrollar metodologías como base para la producción de objetos de design sostenible a partir de la reinterpretación de técnicas tradicionales de los indígenas Zenú, una comunidad que subsiste hasta el día de hoy en la región Caribe al norte de Colombia (departamentos de Sucre, Córdoba y norte de Antioquia), y que mantiene gran parte de sus valores culturales, asociados a la producción de artesanías.

Se proponen en el estudio generar nuevas posibilidades de desarrollo de productos a partir de la reinterpretación de los conocimientos de las comunidades indígenas Zenú, en la construcción de diálogos entre design y artesanado, que vayan más allá del desarrollo de trabajos colaborativos, enfocados en el sentido de la creación de sinergias que hagan posible el empoderamiento de las comunidades periféricas desde un nuevo posicionamiento tecnológico, el cual fue truncado por procesos de colonización y pos-colonización que desencadenaron el sistema mundo vigente que ha determinado la historia de América latina (QUIJANO, 2000).

Cada vez más se realizan en la actualidad y en diferentes contextos, investigaciones en las que se exploran diálogos significativos entre design y artesanado, como por ejemplo, los estudios desarrollados por Barbosa (1983), Yair et al. (2001), Tung (2012), Pacheco et al. (2013), Campbell (2014), Alexandre et al. (2015), Saskia (2015), UNESCO (2015), Medonça et al. (2017), entre otros, a partir de los cuales se puede entender la integración design artesanado, como una estrategia prometedora que además de estimular la innovación y el desarrollo de productos diferenciados, permite explorar nuevas posibilidades de desarrollo para todos los actores involucrados.

Con relación a la creciente demanda en el mercado mundial para la utilización de tableros de madera elaborados con diferentes residuos agroindustriales, se puede observar que han surgido un gran número de estudios enfocados en la identificación de las potencialidades de diferentes fibras y partículas lignocelulósicas para la producción de tableros como alternativa a la utilización de maderas finas y de lento crecimiento.

Recientemente se ha explorado sobre el potencial que ofrece la Caña flecha o Cana - brava como es conocida en Brasil (Gynerium sagittatum), como material alternativo para el desarrollo de nuevos productos forestales innovadores que puedan ser una opción competitiva a la utilización de la madera (figura 01).
Figura 01 - Caña-flecha, Cana-brava, Gynerium sagittatum.

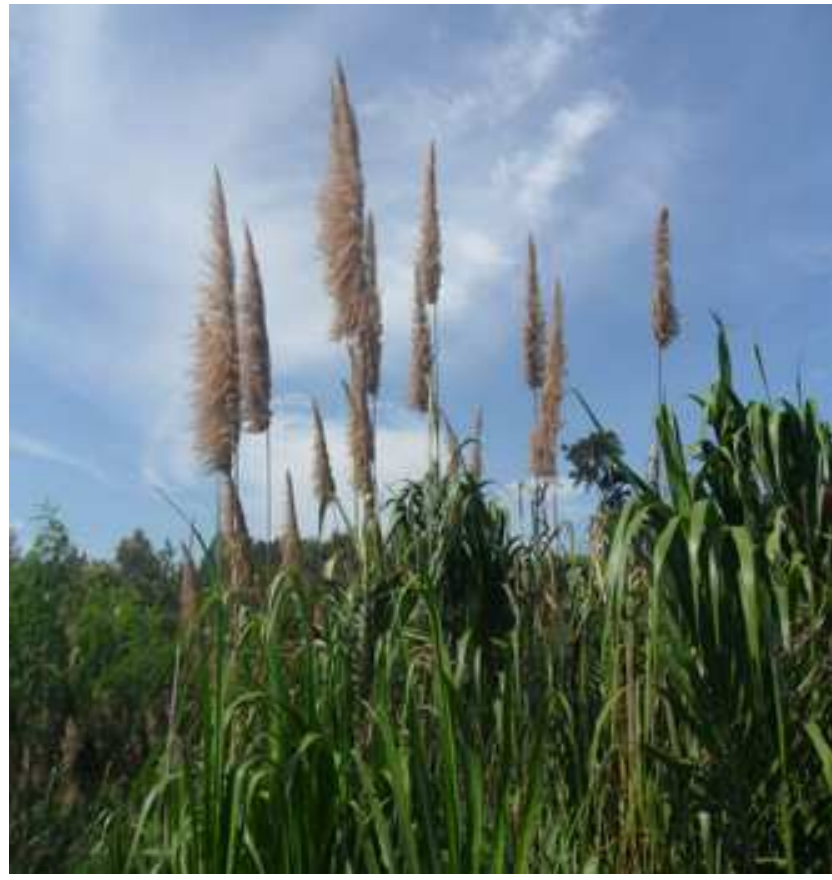

Fuente: los autores.

Entre los diversos estudios, es interesante el trabajo llevado a cabo por Contreras y Owen de Contreras (1997), para el desarrollo de elementos estructurales laminados, tipo parallam, con tiras de Gynerium sagittatum y adhesivo fenol -formaldehido. Los investigadores realizan un procesamiento mecánico de los tallos de Gynerium sagittatum, obtienen tiras con espesores promedios de $3 \mathrm{~mm}$ largo $3100 \mathrm{~mm}$ y ancho aproximado de $30 \mathrm{~mm}$. El estudio encuentra valores de ensayos menores a las exigencias de la norma utilizada como referencia, describen una relación entre la calidad de la línea de cola y los valores bajos obtenidos, tomando como causa negativa la mala humectación de la cola en las tiras cuando coinciden con la cara que presenta la cutícula impermeable externa de la Gynerium sagittatum (CONTRERAS E OWEN DE CONTRERAS, 1997).

Otro estudio importante se identifica en el trabajo desarrollado por Contreras et al. (1999), en el cual proponen la elaboración de tableros aglomerados con partículas de Gynerium sagittatum y adhesivo urea-formaldehído. Los autores desarrollaron ensayos con tableros al 10 y $13 \%$ de resinosidad, obtienen un mejor desempeño en las pruebas de laboratorio, para los conformados con un $13 \%$ de resina. Encuentran deficiencias en el proceso de producción de las partículas para la conformación del tablero. En el trabajo se relaciona la baja resistencia de los tableros a las características del molino y la mala calidad en el afilado de las cuchillas, que 
ocasionaron que gran parte de las partículas se convirtieran en polvillo, lo cual posiblemente produce falta de traba entre las partículas en la conformación del tablero.

Puede atribuirse esta baja resistencia a que en los tableros elaborados bajo estos parámetros existió una mala calidad en la producción de las partículas con las cuales se hicieron los mismos, debido a la irregularidad de formas y tamaños obtenidos y analizadas de las probetas ensayadas las cuales se obtuvieron en el molino del laboratorio de pulpa y papel del LNPF, el cual está diseñado para la realización de astillas de bloques de madera sólida. Se pudo determinar que por la mala calidad del afilado de las cuchillas, la gran velocidad de las revoluciones del portacuchillas ocasionó que se volvieran gran parte de las partículas en polvillo y otras de mediana e irregular tamaño ocasionando una posible falta de traba entre partículas al momento de elaborar los tableros. (CONTRERAS ET AL., 1999, p. 133).

Aunque Contreras et al. (1999) Concluyen que los tableros desarrollados pueden ser aplicados a una multiplicidad de usos, también identifican debilidades en la estructura anatómica propia de la Gynerium sagittatum, como factor determinante de la baja resistencia, debido a que esta presenta dos zonas bien definidas, una interna parenquimatosa, y otra externa esclerenquimatosa, lo que define la calidad del material lignocelulósico de la Gynerium sagittatum (CONTRERAS ET AL., 1999).

En otro estudio desarrollado por Contreras et al. (2008), en el que se determinan los niveles de sostenibilidad en el proceso de fabricación de tableros con Gynerium sagittatum; utilizan un método propio que desarrollaron denominado "método ACV-Coclowen". Identifican en ese trabajo los indicadores de sostenibilidad negativos más significativos del proceso en las etapas de adquisición del adhesivo utilizado, la transformación de las cañas en partículas, tamizado y clasificación de partículas, secado de partículas, encolado, la conformación del tablero a través de presión / calor, y el dimensionamiento final del tablero.

Los autores sugieren en sus conclusiones hacer uso de adhesivos más ecológicos, la aplicación de normas de seguridad industrial en la etapa de encolado para disminuir los riesgos de toxicidad al operario; y para el diseño de la industria, utilizar residuos de madera para alimentar las calderas en el proceso de secado de partículas (CONTRERAS ET AL. 2008).

Una investigación reciente de Gallego et al. (2014) (a partir del trabajo de GALLEGO, 2014), también con la intención de mejorar los indicadores de sostenibilidad de los procesos de producción de tableros de partículas de Gynerium sagittatum, propone el desarrollo de tableros aglomerados, sin uso de adhesivos sintéticos. Los autores explican el potencial presente en la lignina que compone Gynerium sagittatum como alternativa para reemplazar los adhesivos en tableros aglomerados. El estudio propone procesos de pre tratamiento como el steam explosion, para el desarrollo de tableros de partículas de alta densidad de Gynerium sagittatum prensadas sin uso de aditivos. Entre sus resultados identifican una relación entre los mejores comportamientos a las pruebas mecánicas, y la severidad del pre tratamiento utilizando steam explosión (GALLEGO ET AL., 2014).

Por otra parte un estudio que discute la interacción entre Design, artesanado e innovación social, desarrollado por Medonça et al. (2017), a partir a partir del proyecto de intervención realizado por el Laboratório Imaginário Pernambucano en el distrito de Ponta de Pedras, municipio de Goiana, estado de Pernambuco, Brasil; resulta muy interesante debido al objetivo de valorar la identidad local y promover la calidad de vida sostenible de las comunidades.

La metodología utilizada en el trabajo, estimula el reconocimiento del saber popular, específicamente en este caso en torno a la cestería con Gynerium sagittatum (Canabrava), consolidando la actividad artesanal en la región como fuente de renta y trabajo para la comunidad. Entre los aspectos positivos que destacan en sus conclusiones, se encuentra el mejoramiento de las condiciones técnicas de procesamiento, tanto en la extracción de la materia prima, así como el desarrollo de maquinaria adecuada para facilitar la producción de piezas (MEDONÇA ET AL., 2017).

Con relación al conocimiento de los indígenas Zenú de Colombia, y su larga tradición en cuanto al procesamiento de la Caña- flecha, se puede encontrar parte de ese conocimiento ancestral, recopilado en la Norma Técnica Colombiana NTC 5714 (ICONTEC, 2009), la cual establece criterios de sostenibilidad para el desarrollo de productos artesanales con fibras de Caña - flecha.

La norma NTC 5714 describe y establece indicadores de sostenibilidad en prácticamente todo el proceso de producción artesanal; desde el cultivo de la Caña flecha hasta la confección, elaboración y rotulado de los productos artesanales. Entre los procesos que describe la norma, se destacan aquellos que hacen parte del conocimiento ancestral de las comunidades indígenas Zenú: despaje y desvarite, raspado, blanqueado, ripiado, tinturado y trenzado de las fibras de Caña Flecha (ICONTEC, 2009).

El "despaje desvarite" es un proceso de reducción de 
la hoja para dejar la nervadura central, en la que se encuentra la fibra principal la cual se procesa y se obtiene toda una serie de artesanías trenzadas.

El "despaje y desvarite" consiste en retirar la parte ancha de la hoja dejando solo la nervadura central como parte aprovechable para la elaboración de artesanías. Este proceso se realiza con herramientas afiladas, además de exigir su realización con elementos mínimos de seguridad, limpieza personal y disposición de residuos, los cuáles pueden aprovecharse como alimento para animales o abonos orgánicos (ICONTEC, 2009, p. 8).

En el "raspado" se retira la capa vegetal de la nervadura central a través de un proceso manual de beneficiamiento con herramientas menores:

Proceso en el cual se retira la capa vegetal de la vena de la hoja, empleando un cuchillo de filo medio. El procedimiento se realiza colocando la zapatilla en la pierna, sobre la cual se coloca la fibra y con el cuchillo se hace el raspado de las hojas, este procedimiento se repite cuantas veces sea necesario, hasta que la fibra quede completamente suave. Este proceso es determinante para la calidad de la fibra. (ICONTEC, 2009, p.9).

Es interesante de este proceso, que a pesar de ser una técnica ancestral del procesamiento de la Caña flecha por parte de la comunidad indígena Zenú de Colombia, es muy similar con otros procesos para el beneficiamiento de la Caña flecha por parte de artesanos de Brasil (ver COÊLHO, 2013), así como procesos de beneficiamiento del bambú para la producción de artesanías por parte se artesanos en diferentes contextos, especialmente en países asiáticos.

El proceso de "blanqueado" consiste en aclarar las fibras para su posterior uso:

Se realiza en agua de caña agria. Para ello se dispone suficiente agua limpia en un recipiente según la cantidad de fibra a blanquear. Se pela la caña agria, quitándole la concha a la vara con un machete. Se machaca, pila o tritura la caña pelada sobre una superficie dura para que suelte el jugo y luego se exprime este en el agua. Se revuelve el agua se depositan los manojos de caña flecha a blanquear, poniendo encima el bagazo de la caña agria para mantenerla sumergida adicionarle ácido cítrico, dejándola en el agua de caña agria durante $12 \mathrm{~h}$ o una noche.

Al día siguiente se saca la caña flecha, se lava bien con la misma agua, evitando que se adhieran pedazos del bagazo, se pone al sol y sereno durante $2 \mathrm{~d}$ o $3 \mathrm{~d}$, hasta que adquiera su color natural. Hay que evitar sobre exponerla al sol, ya que se vuelve amarilla o se ensucia con el polvo. En caso de que falte caña agria, se puede usar naranja o limón (ICONTEC, 2009, p.11).

El proceso de "ripiado" consiste en dividir la fibra en fragmentos más delgados, lo cual determina la calidad del trenzado posterior:

Se hacen fibras delgadas o "pencas" de acuerdo a la calidad de la trenza que se vaya a tejer. Es importante ripiar todo el lote de la caña flecha que se está preparando de una sola vez, ya que pueden quedar pencas de diferente grosor el trenzado no quedará uniforme (ICONTEC, 2009, p.12).

A partir de las diferentes referencias, y teniendo en cuenta todo el conocimiento ancestral implícito en las técnicas artesanales indígenas, es posible identificar aspectos por mejorar con relación a la sustentabilidad de los procesos de producción de nuevos productos forestales a partir de Caña -flecha, Gynerium sagittatum. Se propone superar las debilidades identificadas en los diferentes estudios, aplicando en la elaboración de los tableros, algunos procesos que reinterpreten las técnicas tradicionales indígenas, de esta forma generar unos mejores indicadores de sostenibilidad en la producción, por lo cual también es necesario tener en cuenta, el tipo de adhesivos utilizados y el tipo de recursos energéticos utilizados en el proceso de producción, entre otros aspectos.

\section{MATERIALES Y MÉTODOS}

La investigación en curso de la que hace parte este trabajo consiste en un estudio experimental, con abordaje deductivo de tipo cuantitativo, desarrollado en tres fases a saber: fase 1 descriptiva - exploratoria (exploración sobre las propiedades del material y las técnicas a implementar); fase 2 experimental (análisis del material a partir Gynerium sagittatum, re interpretando técnicas tradicionales de los indígenas Zenú); fase 3 proyectual (design de producto de mobiliario con material a partir de Gynerium sagittatum.

Los resultados preliminares que se muestran en este artículo hacen parte de la primera y segunda fase del estudio.

La recolección del material de Gynerium sagittatum (Caña- flecha) para el desarrollo de los cuerpos de prueba para los ensayos de resistencia se realizó en la ciudad de Agudos, São Paulo, Brasil.

La metodología para el procesamiento de los cuerpos de prueba de material aglomerado a partir de partículas de Gynerium sagittatum se desarrolló en el Laboratorio Didáctico de Materiales y Prototipos de la UNESP (LDMP) 
campus Bauru, Brasil, con los siguientes procesos:

Se benefició el material de forma manual tomando como referencia la técnica del "raspado" de la comunidad indígena Zenú, aplicándola en este caso a los colmos para retirar la mayor cantidad de material lignocelulósico de la capa interna de Gynerium sagittatum.

En primera medida se elimina la hoja que recubre el colmo, dejando al descubierto el material a trabajar. Este proceso se desarrolla con herramientas menores sean estos cuchillos o machetes (figura 02).

Figura 02 - Eliminación de la hoja que recubre el colmo de Gynerium sagittatum.

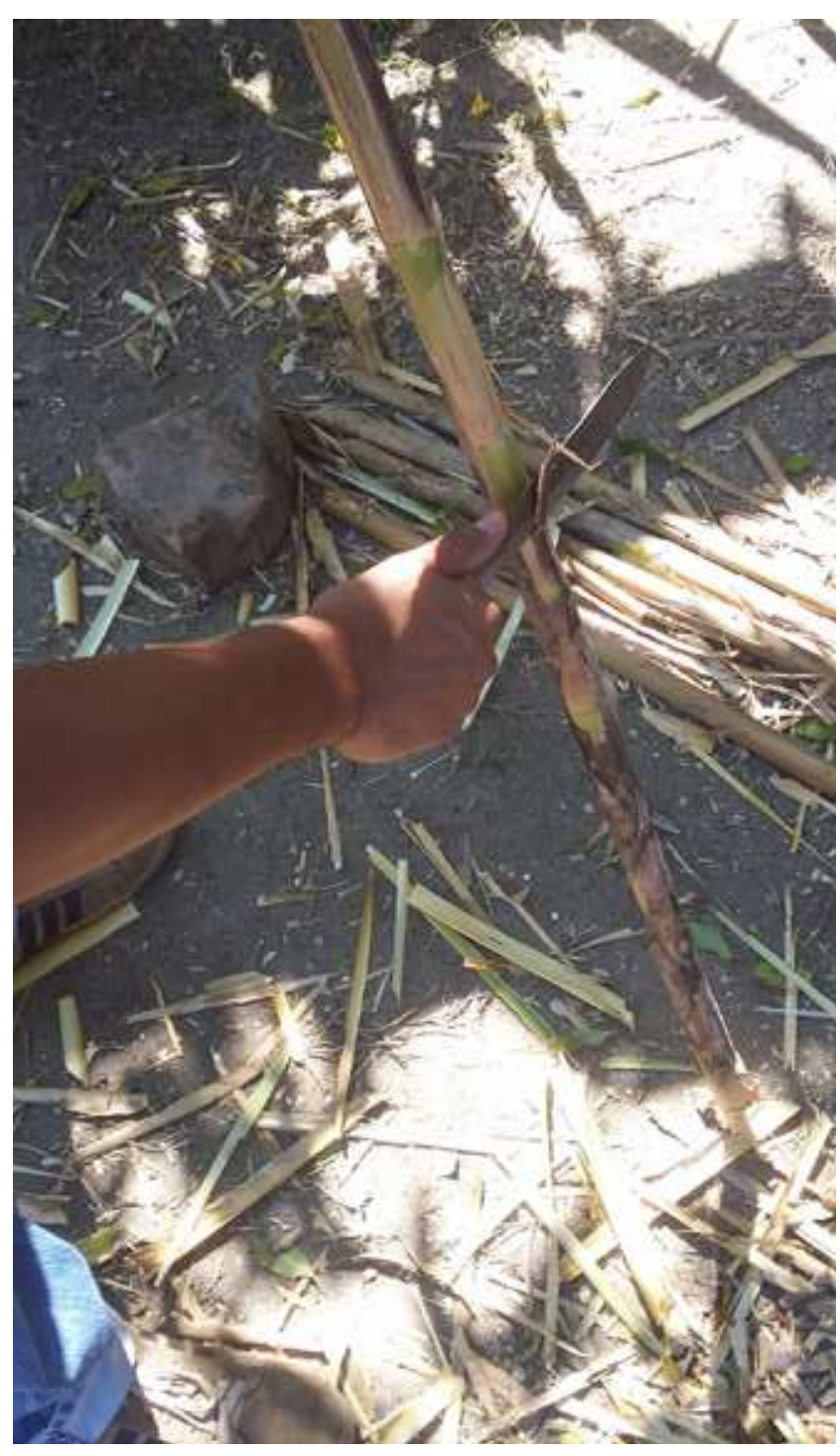

Fuente: los autores

Luego se realiza un corte transversal con sierra manual para definir la longitud de los colmos que serán trabajados, en este caso se definió como longitud $1.00 \mathrm{~m}$.

Después se realizó un corte longitudinal para dividir el colmo en 4 partes utilizando cuchillo tipo estrella. Este procedimiento permite exponer el material lignocelulósico de las capas interiores del colmo (figura 03).

Figura 03 - Cuchillo tipo estrella para corte longitudinal.

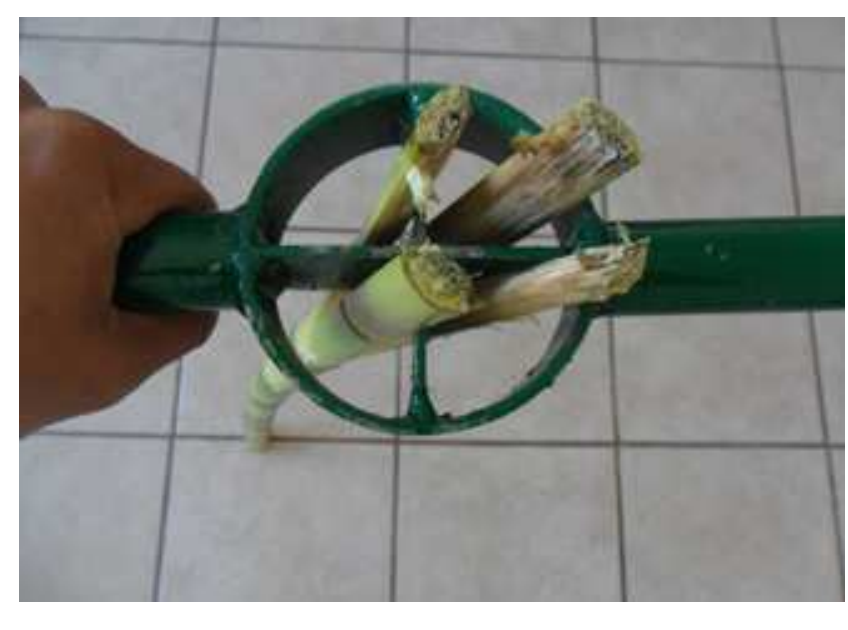

Fuente: los autores

Se realizó una inmunización de las piezas con octaborato dissódico tetrahidratado por inmersión, en una proporción 1:20 durante 4 horas. El material se dejó secar a la sombra en un lugar ventilado, seco protegido de la lluvia y la humedad.

Para el beneficiamiento, se trabaja el material de forma manual retirando primeramente con cuchillo gran parte del material lignocelulósico de la capa interna, el proceso es ayudado con el uso de martillo para retirar de forma homogénea el material. La pieza se trabaja en forma vertical golpeando el cuchillo de arriba hacia abajo, se logra obtener una tira regular de 1.5 - $2 \mathrm{~cm}$ de ancho y grosor variable alrededor de 3 a $5 \mathrm{~mm}$ (figura 04).

Figura 04 - Corte longitudinal para eliminar la capa interna.

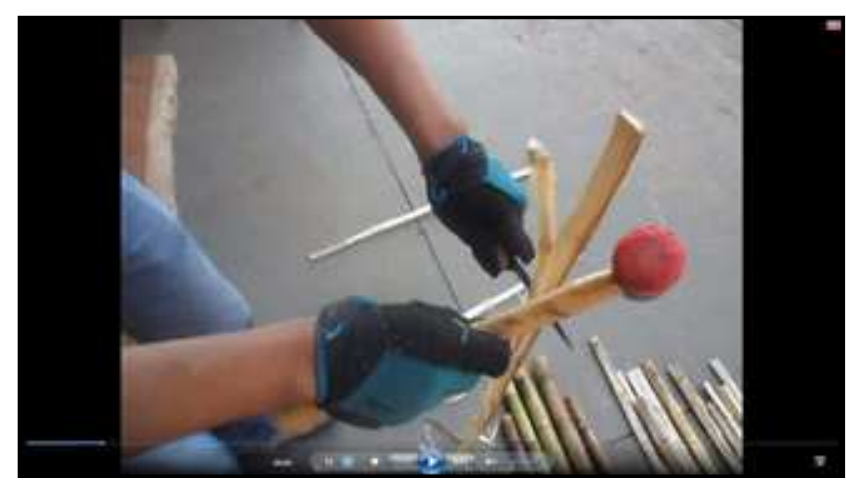

Fuente: los autores

Después tomando como referencia a técnica de "raspado" de las hojas de Gynerium sagittatum, se 
aplica un beneficiamiento al colmo, "raspando" con el cuchillo removiendo las capas internas y externas generando virutas de diferentes tamaños. De cada metro de colmo son retirados de forma manual aproximadamente $105 \mathrm{~g}$ de partículas (figura 05).

Figura 05 - "Raspado" manual de la tira de Gynerium sagittatum.

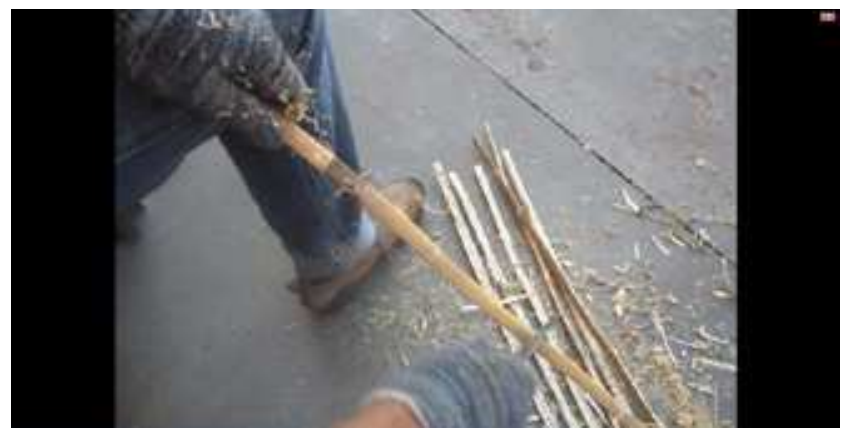

Fuente: los autores

Producto del proceso de "raspado" se pueden identificar dos tipos de partículas, una alargada y grosor variable de buen aspecto y consistencia proveniente de las capas internas, otra de delgada y enroscada de poca consistencia proveniente del "raspado" en la capa externa (figura 06).

Figura 06 - Dos tipos de partículas producto del beneficiamiento manual.

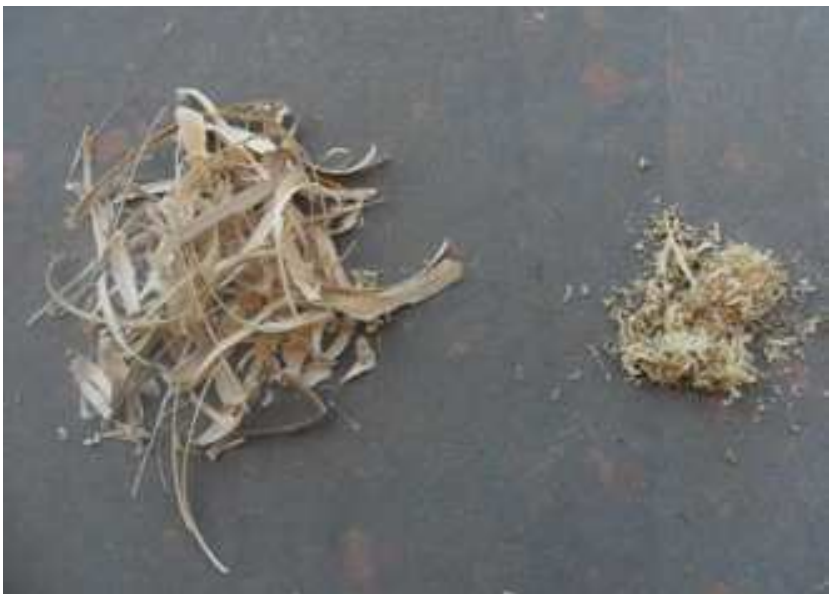

Fuente: los autores

Se exploró la posibilidad de mejorar el rendimiento del proceso utilizando herramientas mecánicas tipo cepilladoras para retirar rápidamente el material de la capa interna y a su vez producir partículas de dimensiones más homogéneas para ser utilizadas en el desarrollo de materiales forestales tipo panel. Se utilizaron para mejorar este proceso en el estudio 2 tipos de máquinas: cepillo de banco marca Baldan DPC-3, cepillo eléctrico manual marca
Stanley STPP7502- B3 $120 \mathrm{~W}$ - 60 Hz, las cuales pueden ser relativamente de fácil adquisición para una posible transferencia tecnológica en comunidades carentes.

Se observó que las partículas producto del mejoramiento con cepilladoras eléctricas son más homogéneas (figura 07), se identifica como aspecto positivo el hecho de optimizar el proceso de retirada del material lignocelulósico. Se encuentra que las partículas producto del beneficiamiento manual aplicado a la capa interna son de mayor tamaño y consistencia. Se piensa que estas partículas podrían generar una mayor traba y por consiguiente mayor resistencia del panel (figura 08).

Figura 07 - Partículas producto del mejoramiento del proceso con cepilladora de banco.

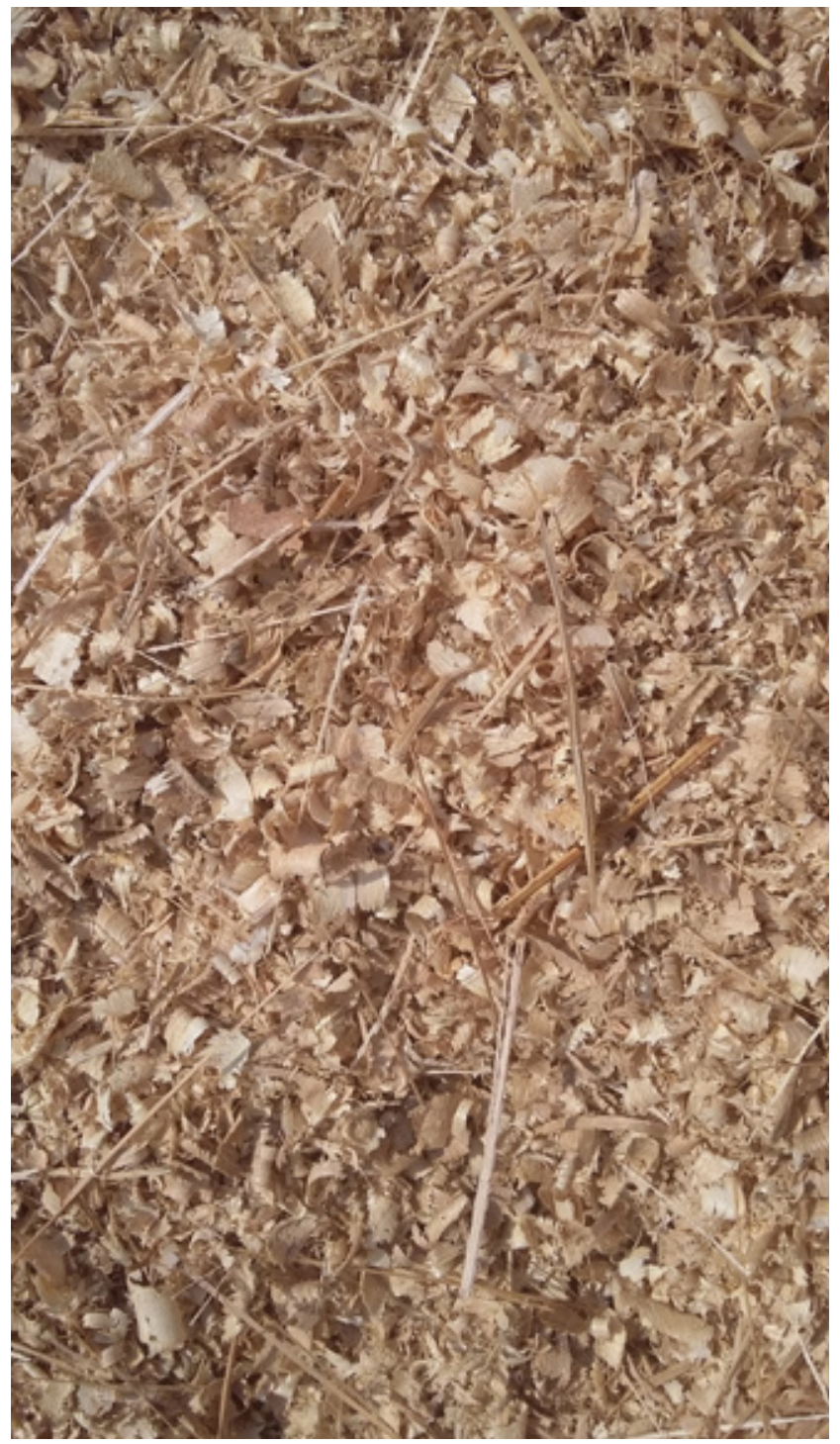

Fuente: los autores 
Figura 08 - Partículas producto del beneficiamiento manual tipo "raspado”.

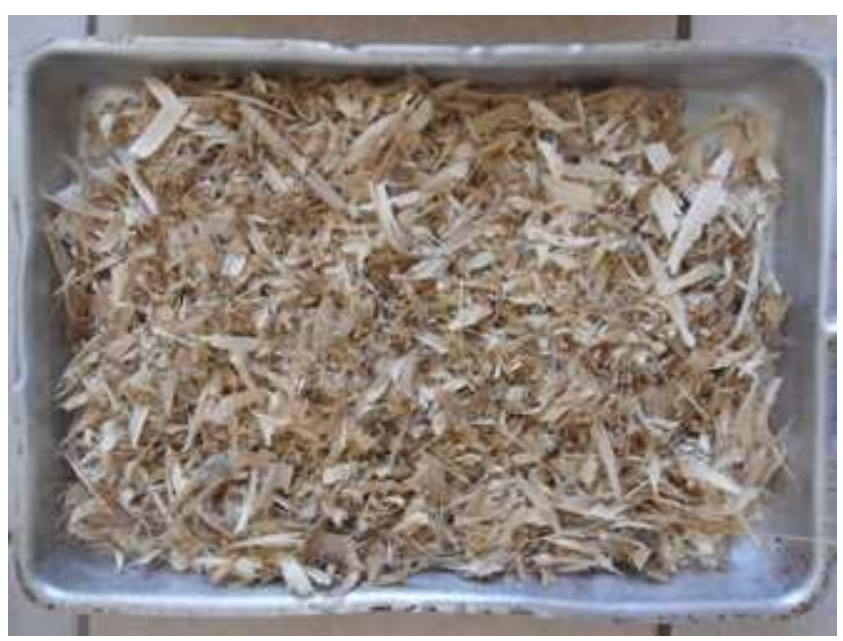

Fuente: los autores

Se decidió utilizar todas las lascas producto del proceso de "raspado" manual para la conformación del colchón, con la finalidad de explorar la resistencia del tablero elaborado con material heterogéneo y sin clasificar, privilegiando el trabajo manual con el menor uso de maquinaria posible, pensando en un mayor aprovechamiento de las partículas y una futura transferencia a comunidades periféricas carentes.

El colchón se conformó de forma manual, utilizando como adhesivo resina poliuretana Bi componente AG201 a base de óleo de mamona de la industria Kehl polímeros, con un índice de resinosidad del 15\% con proporción 1:2. Se pre prensaron 5 capas de partículas de Gynerium sagittatum con 75 gramos cada una, con una dimensión de $27 \times 27 \mathrm{~cm}$, que luego fueron prensadas en una prensa manual durante 24 horas (figuras 09, 10, 11).

Figura 09 - Preparación del colchón con partículas de Gynerium sagittatum (pre prensado).

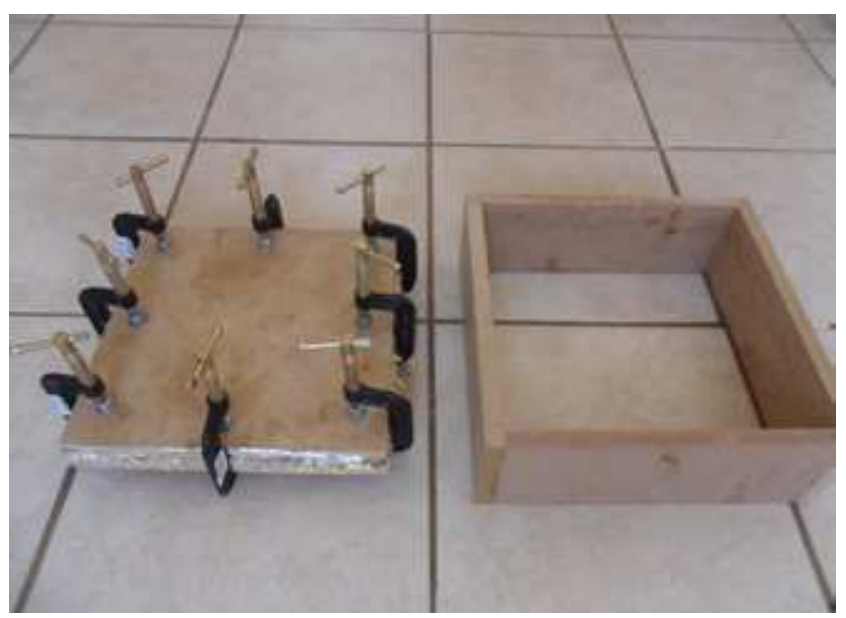

Fuente: los autores
Figura 10 - Preparación del colchón con partículas de Gynerium sagittatum.

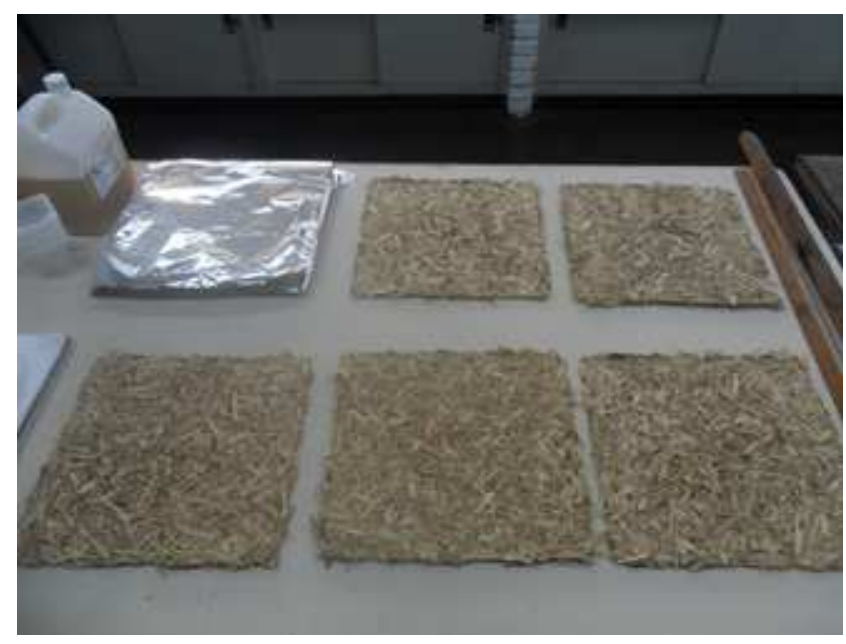

Fuente: los autores

Figura 11 - Prensa manual para la consolidación del panel.

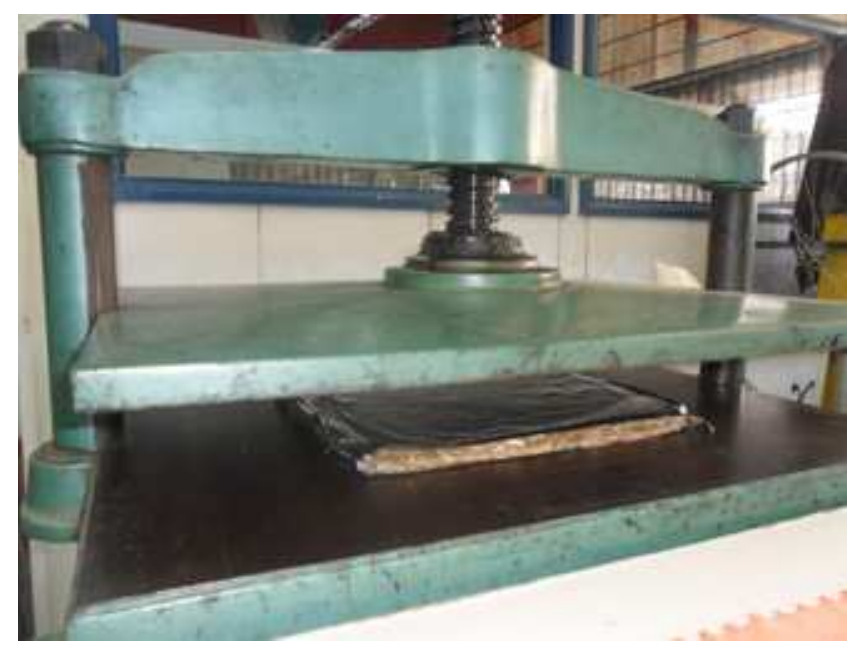

Fuente: los autores

Para la elaboración de los cuerpos de prueba se realizó un corte utilizando sierra de cinta marca Baldan, potencia 2,0cv trifásica, para el dimensionamiento y procedimientos de ensayo se tuvo en cuenta la norma ABNT NBR 14810-3 de 2002. Se realizaron pruebas para establecer el comportamiento de las chapas de partículas sometiéndolas a ensayos destructivos y no destructivos como: densidad, humedad, absorción de agua, tracción perpendicular, tracción paralela, flexión estática, compresión longitudinal. Estos ensayos fueron realizados en maquina universal de ensayos DL 30.000 marca EMIC, propiedad de la Facultad de Ingeniería, FEB, UNESP, campus Bauru.

\section{RESULTADOS Y DISCUSIÓN}

Para la realización de las pruebas se elaboraron 6 
tableros de partículas de Gynerium sagittatum de $27 \times 27$ $\mathrm{cm}$, de los cuales se extrajeron los cuerpos de prueba para los ensayos destructivos y no destructivos. Los tableros presentaron buen aspecto y facilidad para el corte en las diferentes dimensiones (figuras 12 y 13).

Figura 12- Tableros de partículas de Gynerium sagittatum.

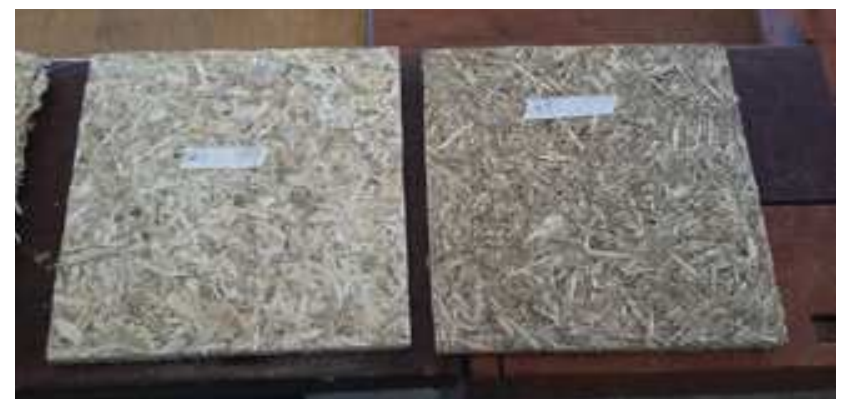

Fuente: los autores

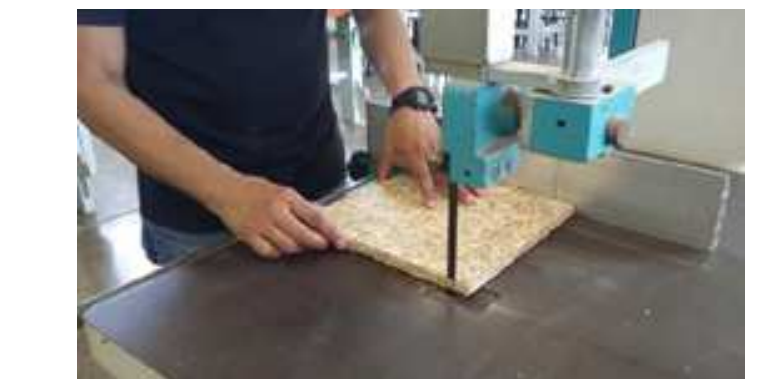

Fuente: los autores

A pesar de la facilidad del corte se notó desde la conformación de los cuerpos de prueba que, si bien los cuerpos de prueba estaban consolidados, estos desprendían muchas partículas, indicando preliminarmente problemas en la unificación de las partículas que conforman el tablero. Algunas de las piezas luego del corte presentaron una gran variedad dimensional, evidenciando grandes diferencias en la compactación producto del prensado manual (figura 14).

Figura 14 - Diferencias en la consolidación de los cuerpos de prueba.

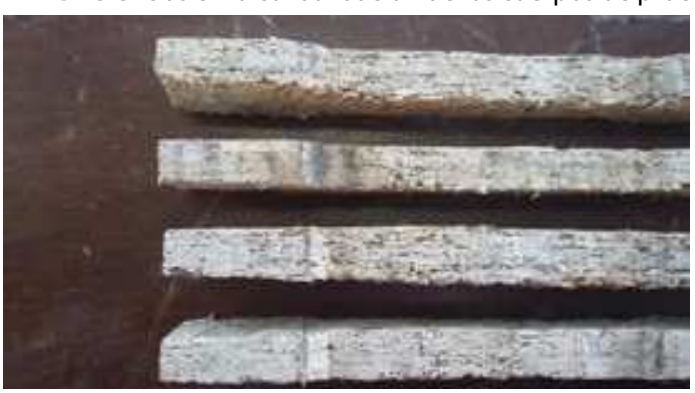

Fuente: los autores
Los resultados preliminares muestran aspectos interesantes a ser rescatados del proceso, aunque el material se mostró muy poco eficiente respecto a algunos esfuerzos, sobre todo tracción perpendicular en el que se obtuvo registros muy bajos.

Tabla 01: Comparativo resultados preliminares com referencia a la norma NBR14810

\section{Resultados con referencia a la norma ABNT NBR 14810 de 2002}

\begin{tabular}{|c|c|c|c|c|c|}
\hline & $\begin{array}{l}\text { Tracción } \\
\text { perpen- } \\
\text { dicular } \\
\mathrm{MPa}\end{array}$ & $\begin{array}{l}\text { Flexión } \\
\text { estáti- } \\
\text { ca MPa }\end{array}$ & $\begin{array}{c}\text { Hincha- } \\
\text { miento } \\
24 \mathrm{Hs}\end{array}$ & $\begin{array}{l}\text { Tracción } \\
\text { paralela } \\
\text { Kgf/cm2 }\end{array}$ & $\begin{array}{l}\text { Compre- } \\
\text { sión } \\
\text { Longitu- } \\
\text { dinal } \\
\text { Kgf/cm2 }\end{array}$ \\
\hline $\begin{array}{l}\text { Tableros } \\
\text { de } 14- \\
-20 \mathrm{~mm} \\
\text { (NBR } \\
14810)\end{array}$ & 0,35 & $\begin{array}{c}16 \\
\mathrm{MPa}\end{array}$ & $\begin{array}{c}8 \% 2 \mathrm{hs} \\
-22 \% \\
24 \mathrm{hs}\end{array}$ & - & - \\
\hline media & 0,01 & 1,47 & $\begin{array}{l}27 \%- \\
24 \text { hs }\end{array}$ & 5,41 & 5,54 \\
\hline máximo & 0,03 & 3,54 & - & 12,41 & 9,50 \\
\hline Mínimo & 0,00 & 0,85 & - & 0,74 & 2,19 \\
\hline $\begin{array}{l}\text { Desv } \\
\text { Padrón }\end{array}$ & $\begin{array}{l}0,0104 \\
5 \mathrm{Mpa}\end{array}$ & $\begin{array}{l}0,7603 \\
\mathrm{MPa}\end{array}$ & - & $\begin{array}{l}3,157 \\
\mathrm{~K} \mathrm{~g} \mathrm{f} \mathrm{/} \\
\mathrm{cm} 2\end{array}$ & $\begin{array}{l}2,713 \\
\mathrm{Kgf} / \mathrm{cm} 2\end{array}$ \\
\hline $\begin{array}{l}\text { c o e f. } \\
\text { variaci- } \\
\text { ón }\end{array}$ & $92,42 \%$ & $51,70 \%$ & - & $58,31 \%$ & $48,93 \%$ \\
\hline
\end{tabular}

Fuente: los autores

Al observar los bajos registros obtenidos en la prueba de tracción perpendicular, en el que el mayor registro alcanzado fue 0,03474 MPa y el mínimo 0,0007999 MPa, con un coeficiente de variación del $92,42 \%$ en 11 cuerpos de prueba, hace pensar que estos registros tan bajos y con tan alta variación entre los distintos cuerpos de prueba, 
están relacionados con la forma escogida para la conformación manual de colchón. Las diferencias evidentes en la compactación de los cuerpos de prueba puede ser un factor determinante en los bajos resultados obtenidos.

Como resultado preliminar de la primera fase y parte de la segunda fase del estudio se desarrolló un proceso con indicadores más sostenibles en la elaboración de tableros de partículas de Caña- flecha, aplicando durante el proceso la re interpretación de la técnica del "raspado" usada por los indígenas Zenú de Colombia para el beneficiamiento inicial de los colmos y la obtención de las partículas con la que se elaboró el colchón.

En las investigaciones de Contreras y Owen de Contreras (1997), y Contreras et al. (1999), se identifica como una debilidad a superar, la composición física de la Caña-flecha específicamente hablando de la diferencia entre las capas internas y externas del colmo.

Como alternativa para superar la debilidad que describen los investigadores, se encuentra valor en las técnicas artesanales para el procesamiento de la Cañaflecha desarrolladas por los indígenas Zenú, las cuales al ser aplicadas en las tiras de Gynerium sagittatum como beneficiamiento de las piezas, permite la eliminación de gran parte de la capa exterior esclerenquimatosa, evita que la fibra se convierta en polvillo y reaprovecha gran parte de la capa interna como fuente de partículas y fibras para ser usadas en la elaboración de productos sostenibles por su alto contenido lignocelulósico.

También Contreras et al. (2008), como conclusiones de su trabajo para determinar niveles de sustentabilidad en los procesos de fabricación de tableros de Gynerium sagittatum, encuentran entre otros, indicadores negativos en relación al adhesivo, la transformación de los colmos en partículas y la conformación del tablero. Como una alternativa a los indicadores de sustentabilidad negativos en relación al adhesivo, se trabajó aquí con resina poliuretana a base de Óleo de Mamona (KHEL), el cual es un adhesivo de menor impacto ambiental y se piensa que cumple a satisfacción sus funciones de consolidación de las partículas a pesar de ser trabajado en frio.

Con relación al gasto energético en el proceso, se piensa que Gallego et al. (2014), en busca de lograr una disminución en el consumo de adhesivos, utilizan procesos que generan un alto consumo de energía como el steam explosión, por lo cual la técnica artesanal implementada, resulta interesante al ser utilizada en conjunto con un adhesivo de bajo impacto ambiental como la utilizada en este estudio.

Como aspectos a mejorar del proceso desarrollado se piensa modificar algunos aspectos en la conformación del colchón, sobre todo en el proceso de prensado manual, optar por un prensado con una prensa hidráulica que pueda generar mayor fuerza de prensado y también garantizar la uniformidad en el prensado de tablero, y a su vez sea un equipo de fácil adquisición para una eventual transferencia tecnológica en comunidades periféricas carentes.

También se piensa que es importante la homogenización del material de partículas para la elaboración del colchón, lo cual se puede conseguir fácilmente utilizando tamices. El material residual del tamizaje, puede ser sometido a un proceso de corte en molinos para obtener unas partículas menores que puedan ser usadas en las capas exteriores mejorando la presentación del tablero y de esa forma no se generen desperdicios.

\section{CONCLUSIONES PRELIMINARES}

Como conclusiones preliminares de la fase exploratoria del estudio, se encuentran aspectos positivos para generar indicadores de sostenibilidad en la utilización de técnicas artesanales para los procesos de beneficiamiento inicial de los colmos y obtención de partículas en la elaboración de tableros de Gynerium sagittatum.

Se observó que al aplicar el "raspado" a la parte externa del colmo, se elimina la capa esclerenquimatosa y se generan virutas delgadas y alargadas de diferentes dimensiones. Este proceso se piensa netamente como un proceso artesanal que puede incluirse a la producción de paneles con Gynerium sagittatum, ya que permite la eliminación de la capa exterior y preserva las tiras para su uso posterior en diferentes tipos de procesos que generen nuevos materiales forestales, sin la necesidad de usar cuchillas mecánicas que conviertan en astillas el colmo.

Se observa que la técnica del "raspado" para la capa interna del colmo de Gynerium sagittatum puede ser optimizada utilizando maquinaria simple con poco gasto energético, que ayude a un mayor rendimiento y que permitiría una mayor producción de partículas, sin necesidad que el colmo se convierta en astillas y polvo, generando dos tipos de productos para el desarrollo de paneles, las tiras y las partículas.

Los procesos manuales para la conformación y prensado del colchón deben revisarse y mejorarse utilizando una maquinaria de bajo costo y mínimo gasto energético, que mejoren el comportamiento de los tableros a los esfuerzos mecánicos, pero a su vez mantengan unos buenos indicadores de sostenibilidad en la producción del tablero. Se piensa que una prensa hidráulica puede ser una solución accesible económicamente y con mínimo gasto energético.

Los conocimientos ancestrales inherentes a las 
técnicas artesanales indígenas, son un recurso interesante para ser analizado y aplicado en diferentes procesos en busca de indicadores de sostenibilidad para el desarrollo y elaboración de procesos y productos más sostenibles que puedan ser competitivos en un mercado global que busca cada vez más productos diferenciados por sus indicadores de sustentabilidad y aspectos ligados a la identidad de los lugares donde son producidos.

\section{REFERENCIAS}

ALEXANDRE, C. B., GOMEZ, E. A., VALENTE, A. C. Interdisciplinary relationship between Designer and Craftsman based on Integrated Craft Manufacturing Systems. Procedia Engineering 132, 2015, p. 1089 - 1095

BARBOSA, M. Artesanato, tradição e mudança social um estudo a partir da "arte do oro" de Juazeiro do norte. In: RIBEIRO et al., 1983. O artesão tradicional e seu papel na sociedade contemporânea. Rio de Janeiro, FUNARTE, Instituto Nacional do Folclore, p. 49 - 100.

CAMPBELL $K$, WILLIAM. Crafting Designs: An Archaeology of "Craft" as God Term. Computers and Composition 33 (2014) 50-67

COÊLHO, JACKELINE. Matéria Artesanato Cana Brava Goiana-PE. 2013. Produção e Reportagem Jackeline Coêlho, Imagens Ronaldo Alves, Edição Tony Vasconcelos foi Exibido na Tv Rit Programa Revista do Campo. Disponível online em: https://www.youtube.com/watch?$\mathrm{v}=$ KOhYSh1jEEs acesso janeiro 20 de 2018.

CONTRERAS, WILVER; CLOQUELL, VICENTE; OWEN DE C., MARY. Determinación de los niveles de sostenibilidad del proceso de fabricación de tableros de caña brava (Gynerium sagittatum), a partir del método acv-Coclowen. Revista Forestal Venezolana, Año XLII, Volumen 52(1) enero-junio, 2008. P. 47 - 59.

CONTRERAS, WILVER;OWENDEC., MARYE. Elaboración de un elemento estructural laminado, tipo parallam, con tiras de caña brava Gynerium sagittatum y adhesivo fenol-formaldehído. Revista Forestal Venezolana. Facultad de Ciencias Forestales y Ambientales. Universidad de Los Andes. Mérida, Venezuela 41(1), 1997, 29-36.

CONTRERAS, W.; OWEN DE C., M.E.; GARAY, D.A.; CONTRERAS, Y. Elaboración de tableros aglomerados de partículas de Caña Brava (Gynerium sagittatum) y adhesivo urea-formaldehído. Revista Forestal Venezolana. 43(2) 1999, 129-135.

GALLEGO, GIOVANNA. Tableros sin aditivos a partir de caña flecha (Gynerium sagittatum). Medellín, 2014. Universidad Pontificia Bolivariana. Trabajo de grado en ingeniería Química. Disponível em: <https://repository.upb.edu.co/bitstream/handle/20.500.11912/3148/ Tableros\%20sin\%20aditivos\%20a\%20partir\%20de\%20 la\%20ca\%C3\%B1a\%20flecha.pdf?sequence=1\&isAllowe$\mathrm{d}=\mathrm{y}>$ acesso em: agosto 23, 2017.

GALLEGO, G.; VELÁSQUEZ, J.; QUINTANA, G. Tableros sin aditivos a partir de Gynerium sagittatum. Revista investigaciones aplicadas, Vol. 8, No. 2, jul - dic, 2014. Pp 101 -112 .

ICONTEC. NORMA TÉCNICA COLOMBIANA, NTC 5714. Bogotá. Instituto Colombiano de Normas Técnicas (ICONTEC), 2009. Disponível em: < http://www.minambiente.gov.co/images/normativa/Otros/NTC/2009/ NTC_5714_2009.pdf> acesso em: 25 agosto, 2017.

MEDONÇA, ANA FLAVIA D. F.; RÊGO C., MARIA IZABEL; AIRES D. S., ROSANA. p. 149 - 162. Em: ARRUBLA, AMILTON (Org). Design \& inovação social. Série [designCONTEXTO] Ensaios sobre Design, Cultura e Tecnologia 2. Editora Edgard Blücher Ltda. São Paulo, 2017. P.280. Disponível em: <http://pdf.blucher.com.br.s3-sa-east-1.amazonaws. com/openaccess/9788580392647/completo.pdf> acesso Dezembro de 2017.

PACHECO, J.; BARRERO, G.; GÓMEZ VÁSQUEZ, G. An Eco-Technological Approach to Handcraft Production. Two Cases in the Colombian Caribbean Region. Cuadernos de Desarrollo Rural, 10 (70), 2013, p. 115-129.

QUIJANO, ANIBAL. Colonialidad del poder, eurocentrismo y América Latina. En libro: La colonialidad del saber: eurocentrismo y ciencias sociales. Perspectivas Latinoamericanas. Edgardo Lander (comp.) CLACSO, Consejo Latinoamericano de Ciencias Sociales, Buenos Aires, Argentina. Julho de 2000. p. 246. Disponível em: http://bibliotecavirtual.clacso.org.ar/ar/libros/lander/quijano.rtf acesso julho 9 de 2016.

SASKIA, V. M. Co-ideation of disaster preparedness strategies through a participatory design approach: Challenges and opportunities experienced at Turrialba 
volcano, Costa Rica. Design Studies, 40 (C), 2015, p.218-245.

TUNG, FANG WU. Weaving with Rush: Exploring Craft-Design Collaborations in Revitalizing a Local Craft. International Journal of Design Vol.6 No.3, 2012, p. 71 - 84.

YAIR, K.; PRESS, M.; TOMES, A. Crafting competitive advantage: crafts knowledge as a strategic resource. Design Studies, 22 (4), July, 2001: p. 377-394 